\section{Estressores psicossociais, senso de comunidade e bem-estar subjetivo em crianças e adolescentes de zonas urbanas e rurais do Nordeste do Brasil}

\author{
Psychosocial stressors, sense of community, and \\ subjective wellbeing in children and adolescents \\ in urban and rural areas in Northeast Brazil
}

\section{Estresores psicosociales, sentido de comunidad y bienestar subjetivo en niños y adolescentes de zonas urbanas y rurales del Nordeste de Brasil}

Desirée Pereira de Abreu 1

Ferran Viñas 1

Ferran Casas 1

Carme Montserrat 1

Mònica González-Carrasco 1

Stefania Carneiro de Alcantara 1

\title{
Resumo
}

O objetivo geral do estudo é investigar a relação existente entre eventos estressores cotidianos, senso de comunidade e bem-estar subjetivo em alunos de escolas urbanas e rurais do Nordeste do Brasil, focando nas diferenças por contexto territorial. A amostra está formada por 757 participantes, 495 alunos de escolas urbanas e 262 de escolas rurais, dos 60 e 70 anos (9 a 18 anos) de 21 escolas públicas municipais e estaduais, 13 urbanas e 8 rurais, de 7 municípios do Ceará, Brasil. Os instrumentos utilizados foram: inventário de eventos estressores, escala de satisfação com a vida para estudantes, índice de senso de comunidade e oito índices de satisfação por âmbitos da vida (família, bens materiais, relações, bairro/zona, saúde, tempo, escola, pessoal). Os resultados indicam que os alunos das escolas públicas (desfavorecidos socioeconomicamente) de contextos urbanos estão mais expostos ao estresse cotidiano, pontuam mais baixo em satisfação com âmbitos específicos da vida e senso de comunidade, sendo este último um indicador importante na avaliação do bem-estar da população estudada.

Acontecimentos que Mudam a Vida; Qualidade de Vida; Criança; Adolescente 


\section{Introdução}

Em 2050, sete em cada dez pessoas da população mundial viverão em zonas urbanas 1 . O relatório do Fundo das Nações Unidas para a Infância (UNICEF) sobre a situação mundial da infância em 2012 enfatiza a problemática das crianças em um mundo cada vez mais urbano, alertando que atualmente mais de 1 bilhão de crianças e adolescentes crescem e se desenvolvem em pequenas e grandes cidades, e 43,5\% deles vivem em cidades com mais de 500 mil habitantes. Quando se avalia o bem-estar infantil comparando indicadores objetivos globais de crianças das zonas urbanas e rurais, os resultados do setor urbano tendem a ser melhores. Isso se pode atribuir à grande desigualdade socioeconômica presente, o que faz com que o sofrimento das crianças mais pobres das zonas urbanas seja encoberto pela riqueza daquelas que vivem em zonas favorecidas das cidades 1 .

Uma análise pormenorizada dos dados urbanos põe à mostra as disparidades existentes na sobrevivência, no acesso aos serviços básicos (moradia, saúde, educação etc.) e na proteção social das crianças e adolescentes que vivem em comunidades urbanas pobres e excluídas de direitos; muitas vezes em condições similares ou piores que as crianças pobres das zonas rurais. Tal realidade é agravada quando uma proporção significativa do crescimento da população urbana corresponde justamente às zonas mais empobrecidas e menos planificadas. Um em cada três residentes das cidades vive em condições marginalizadas, sem segurança, em lugares superpovoados e anti-higiênicos, caracterizados pelo desemprego, poluição, tráfico, delinquência, alto custo de vida, baixa cobertura de serviços e competição por recursos 1 .

A qualidade do ambiente físico e social em que crescem as crianças, afeta diretamente seu desenvolvimento, aprendizagem, competência social e comportamento ${ }^{2}$. Crianças são mais vulneráveis às condições adversas que os adultos. O desenvolvimento infantil é afetado por múltiplos fatores, tais como: condição laboral e educacional dos pais, pertencer a grupos minoritários, a famílias pobres, numerosas ou monoparentais, sofrer eventos estressantes etc. ${ }^{3}$. Nas grandes cidades, soma-se a tais problemáticas a exposição permanente à sobrecarga de estímulos informativos presentes nos espaços urbanos e a ausência de contato direto com espaços públicos naturais (zonas verdes e ao ar livre), o que provoca nas crianças uma redução da criatividade, da curiosidade e do envolvimento em relações sociais, produzindo isolamento, estresse, cansaço e fadiga 4 .
Na infância e adolescência, condições familiares de pobreza e exclusão social, e situações cotidianas que envolvem: separação dos pais, doença, conflitos familiares constantes, discussões com autoridade, disputas com amigos, relações com o sexo oposto, exigências escolares, entre outros, podem ser considerados eventos estressores 5. As contrariedades ou hassles 6 , também chamados de acontecimentos menores ou microestressores diários, por serem mais frequentes e menos notórios do que os eventos maiores, induzem menos as ações compensatórias, o que poderia torná-los fontes maiores de estresse diário, quando comparados a eventos vitais. O efeito acumulativo de experiências cotidianas negativas, aparentemente de pouco importância, é considerado um importante preditor de problemas de saúde 7 . Uma pesquisa com alunos de escolas públicas em Pernambuco, Brasil, encontrou associações entre consumo de bebidas alcoólicas e uso de drogas e indicadores de estresse psicossocial 8. Destaca-se, a importância de mensurar não somente a presença de estressores cotidianos, mas também a intensidade atribuída pelas próprias crianças e adolescentes, às experiências negativas vivenciadas 9 .

Em um cenário marcado pelas desigualdades entre os grupos populacionais, e consequentemente por iniquidades na saúde, surge o conceito de determinantes sociais da saúde 10. Esses são caracterizados por circunstâncias econômicas, sociais, culturais e políticas em que vivem e se desenvolvem as pessoas, e que podem estar presentes mesmo antes do nascimento, como é o caso do baixo extrato socioeconômico familiar e do precário nível de escolarização dos pais, fatores que influenciam negativamente na saúde infantil 11,12. Dessa forma, o próprio conceito de saúde, dinâmico e mutável, se transforma em função de condições sociohistóricas e culturais da sociedade que o formula, sendo distinto em cada época. Definida pela Organização Mundial da Saúde (OMS) como bem-estar físico, psicológico e social - no qual influem fatores pessoais, grupais, sociais e culturais em que vive imerso o indivíduo e a coletividade - a saúde deve ser compreendida como um processo no qual o ser humano é capaz de desenvolver suas potencialidades e capacidades buscando sua autorrealização como entidade pessoal e social 13 .

O presente trabalho adotou a definição de bem-estar subjetivo como sinônimo de qualidade de vida ${ }^{14}$, sendo definido como percepções e avaliações do indivíduo sobre a sua própria vida de acordo com aspirações, expectativas, valores culturais, experiências prévias, comparações sociais etc., incluindo apreciações de sua vida em geral, o que se denomina "satisfação vital", e con- 
siderando todos os âmbitos que a compõem em um determinado momento 15 . Quando pensamos em bem-estar subjetivo infantil, o modelo multidimensional de satisfação da vida inclui diferentes âmbitos que influenciam na qualidade de vida de crianças e adolescentes, tais como: bem-estar material, casa e família, educação (escola), relações interpessoais (amigos, vizinhos, colegas), ambiente (bairro ou comunidade), saúde, lazer (uso do tempo livre), participação cidadã e segurança, que em nível pessoal implica sentir-se ouvido e sentir-se seguro 15 .

Na percepção subjetiva do bem-estar e da satisfação com a vida influem tanto aspectos internos ou psicológicos, em suas dimensões afetiva e cognitiva, como as interações externas ou psicossociais com as outras pessoas e com o ambiente 15. O bem-estar subjetivo envolve dimensões como satisfação global com a vida, felicidade e sentimento de pertença 16 . O sentimento psicológico de pertencer a uma comunidade representa o nexo de união entre o individual (psicológico) e o coletivo (social), estando relacionado a apoio social, territorialidade, ambiente social e identidade 17 .

O sentimento de comunidade é definido como o sentimento de pertencer a uma coletividade maior, de ser parte de uma rede de relações de apoio mútuo, disponível e confiável 18. Entre as suas qualidades específicas estão elementos como: fazer parte de, influência, integração, satisfação de necessidades, partilha de vinculações afetivas etc., responsáveis pelo sentimento de que os membros de uma comunidade se importam uns com os outros e buscam satisfazer suas necessidades por meio de um compromisso de união ${ }^{18}$. Tal sentimento tem quatro componentes: percepção de semelhança, interdependência mútua (de relacionamentos e de expectativa), reciprocidade condutual e sentimento de formar parte de uma estrutura social mais ampla, estável e confiável 19 .

O sentimento de comunidade promove o fortalecimento da identidade, da autoconfiança e das relações sociais, contribuindo para o aumento da qualidade de vida e do bem-estar pessoal, combatendo sentimentos de solidão e anonimato 20. Quanto maior a percepção do suporte social recebido, maior é a qualidade das relações sociais, a autoestima, a satisfação vital e, consequentemente, o sentimento de comunidade 20. Pesquisas com adolescentes demostram que a ausência de sentimento de comunidade num bairro, medida pela ausência de influência da escola e da vizinhança na comunidade estudada, leva à solidão e ao isolamento social 21 .

Até o presente momento, não temos conhecimento de estudos anteriores no Nordeste do
Brasil que objetivassem investigar a relação existente entre eventos estressores cotidianos, senso de comunidade e bem-estar subjetivo infantil, focando nas diferenças por contexto territorial.

Buscando preencher a lacuna existente, este trabalho tem como objetivos específicos: (i) identificar os eventos estressores cotidianos de maior incidência e intensidade a que estão expostos crianças e adolescentes de zonas urbanas e rurais do Nordeste do Brasil, segundo a percepção dos participantes; (ii) analisar possíveis diferenças na percepção de estressores cotidianos, no nível de bem-estar e no senso de comunidade entre adolescentes das zonas urbanas e rurais; e (iii) identificar dentre as variáveis estudadas aquelas que estão associadas ao bem-estar subjetivo da população pesquisada, pensando modelos por separado segundo o contexto territorial dos participantes.

\section{Métodos}

\section{Participantes}

A amostra foi composta por 757 participantes, 398 meninos (52,6\%) e 359 meninas $(47,4 \%)$, com idades entre 9 e 18 anos (média $=12,02$ e DP = $1,27)$, dos quais 356 (47\%) cursavam o 6 o ano e 401 (53\%) o 7o ano do Ensino Fundamental, 495 deles $(65,4 \%)$ alunos de escolas urbanas e 262 $(34,6 \%)$ de escolas rurais.

Para selecionar a amostra utilizou-se a técnica aleatória por conglomerados em duas etapas, selecionando-se primeiro a escola e em seguida o ano escolar. De um universo de 3.887 escolas públicas (3.135) e privadas (752) de Ensino Fundamental II, foram selecionadas por sorteio 21 escolas públicas, municipais e estaduais, das quais 13 estavam situadas em zonas urbanas e 8 em localidades rurais, de 7 municípios do Estado do Ceará, Brasil. A estratificação em zona urbana e rural foi proporcional à distribuição das escolas existentes no Ceará em 2013, onde 2.140 escolas eram urbanas e 1.747 rurais.

Os critérios de inclusão das escolas foram: a quantidade de alunos matriculados em cada série (superior a 160 alunos) e a distância geográfica entre o município e a capital do estado (um raio máximo de $100 \mathrm{~km}$ de Fortaleza).

O Estado do Ceará está situado na Região Nordeste do Brasil, ocupando 9,57\% do território desta região e 1,74\% da área total do país. O Ceará é o oitavo estado mais populoso do país e o $3^{\circ} \mathrm{da}$ Região Nordeste, contando com uma população de 8.448.055 habitantes, distribuídos de forma irregular pelas zonas urbanas (6.343.990) e rurais (2.104.065); correspondendo a $15,91 \%$ da popu- 
lação do Nordeste e 4,43\% da população brasileira. O número de crianças e adolescentes entre $5 \mathrm{e}$ 17 anos é de 2.068.833, representando $24,47 \%$ da população total do estado 22 .

\section{Instrumentos}

A Escala de Satisfação com a Vida para Estudantes (Students' Life Satisfaction Scale - SLSS 23) é uma escala do tipo multi-item de livre contexto e autoadministrada, que contém 5 itens para avaliar o grau de satisfação global com a vida de crianças e adolescentes de 8 a 18 anos. Pede-se aos participantes que respondam em uma escala Likert de 5 pontos se estão de acordo ou em desacordo com as seguintes afirmações: minha vida está indo bem, minha vida está indo como eu quero, eu tenho uma vida boa, eu tenho o que quero na vida, as coisas de minha vida são excelentes. Utilizamos a versão brasileira validada por Casas et al. 24. A consistência interna encontrada neste estudo é de 0,86 (alfa de Cronbach).

$\mathrm{O}$ Índice de Senso de Comunidade (Sense of Community Index - SCI 25) é um instrumento autoadministrado que contém 9 itens para avaliar o sentimento de comunidade de crianças e adolescentes de um bairro residencial ou grupo comunitário, por meio de elementos, tais como: pertença, influência, integração, interdependência, participação, satisfação de necessidades, partilha e vinculação efetiva. Esse instrumento foi respondido em uma escala Likert de 5 pontos, variando entre nada de acordo e muito de acordo. Utilizamos a versão brasileira validada por Sarriera et al. 26. Neste estudo, a consistência interna encontrada é de 0,84 .

O Inventário de Eventos Estressores (IEE) 9 é um instrumento composto por 64 itens que avaliam a ocorrência (sim/não) e a intensidade atribuída (escala Likert) de eventos estressores presentes nos âmbitos: pessoal, familiar, escolar e comunitário, segundo a percepção de adolescentes de 12-17 anos. Em estudos anteriores, o instrumento mostrou-se confiável na avaliação de eventos estressores em crianças 27 e adolescentes 9 , independentemente do sexo, idade e tipo de escola.

Seguindo as orientações de Kristensen et al. 9 , realizamos análises separadas para os escores de ocorrência (soma do número total de eventos com base nas respostas "sim") e intensidade atribuída (divisão da soma total dos valores de intensidade pelo número total de eventos). Considerando o objetivo proposto no presente estudo de investigar eventos estressores cotidianos em escolares do Ensino Fundamental de população geral, foram excluídos do inventário original itens referentes a estressores vitais, eventos vi- vidos na etapa final da adolescência e situações relacionadas à população vulnerável em sistema de proteção social; bem como, acrescentados dois itens relacionados à ausência de tempo livre e não acesso às tecnologias digitais. Para a melhor compreensão dos participantes foram realizadas pequenas alterações semânticas em algumas questões. A versão abreviada e adaptada utilizada neste estudo é formada por 30 itens que avaliam a ocorrência (sim/não) e a intensidade atribuída (escala Likert de 6 pontos, 0 a 5) de tais eventos cotidianos, e mostrou-se apropriada em suas propriedades psicométricas na população de estudo, apresentando uma consistência interna de 0,87 para ocorrência e 0,90 para intensidade atribuída.

Índice de Satisfação por Âmbitos da Vida (General Domain Satisfaction Index - GDSI 14). Versão brasileira validada 28 do questionário elaborado pela International Survey of Children's Well-being - ISWeB (http://www.childrensworlds.org) para avaliar oito âmbitos da vida de crianças de 8-12 anos: casa e família, bens materiais, relacionamentos, bairro/zona onde vive, saúde, uso do tempo, escola e satisfação pessoal, compreendendo um total de 33 itens em que os participantes avaliam a sua satisfação por meio de uma escala de 11 pontos (0-10). O instrumento permite ainda calcular o índice de satisfação global baseando-se na média aritmética dos oito índices de satisfação por âmbitos da vida. A consistência interna para os 33 itens é de 0,90.

\section{Procedimentos e aspectos éticos}

Esta investigação está devidamente cadastrada na Plataforma Brasil de Pesquisa Científica, tendo sido submetida à avaliação do Comitê de Ética em Pesquisa da Universidade de Fortaleza, com parecer aprovado (Protocolo no 5.45105). Durante todas as etapas deste trabalho foram respeitados os aspectos éticos e legais de pesquisas que envolvem seres humanos, neste caso, crianças e adolescentes (Resolução no 466/2012 - Conselho Nacional de Saúde).

Após o levantamento de informações oficiais e autorizações de instituições públicas no âmbito da educação em níveis municipal e estadual, selecionamos por sorteio as escolas que cumpriam os critérios de inclusão anteriormente citados, a saber: a quantidade de alunos (mais de 160 matriculados nos 6o e 7ọ anos) e a distância geográfica (raio máximo de $100 \mathrm{~km}$ da capital Fortaleza, Ceará, Brasil)

Os gestores das escolas que aceitaram participar da pesquisa assinaram um Termo de Concordância Institucional. Anteriormente à realização da coleta de dados, foi necessária a autorização 
prévia por escrito tanto dos pais/responsáveis legais (Termo de Consentimento Livre e Esclarecido) como dos próprios alunos participantes (Termo de Assentimento Livre e Esclarecido).

A coleta de dados foi realizada no período de fevereiro a junho de 2014. A administração dos questionários foi realizada na própria escola, no horário escolar, nos turnos manhã e tarde, em dois dias diferentes, com duração aproximada de $1 \mathrm{~h} /$ aula (50 minutos), em grupos de 15 a 30 alunos, separados por ano escolar (6o ou $7 \underline{\text { o }}$ ano). Durante a administração dos questionários estiveram presentes duas pesquisadoras responsáveis, para clarificar dúvidas sobre o preenchimento do mesmo, garantido assim a qualidade dos dados. Os questionários foram respondidos de forma anônima e confidencial, e os resultados são publicados sem que os participantes e as escolas possam ser identificados.

\section{Análises de dados}

Inicialmente, realizamos análises descritivas dos trinta eventos estressores, tanto para a amostra global como segmentando a amostra por zona (urbana e rural), a fim de identificar os estressores com maior prevalência e intensidade atribuída pela população estudada. Posteriormente, realizamos provas de qui-quadrado e testes t de Student, a fim de identificar os eventos cotidianos que apresentavam diferenças com relação à variável contexto territorial, tanto para ocorrência como para intensidade atribuída ao estressor. Foram realizadas ainda, análises do bem-estar (SLSS), senso de comunidade (SCI), satisfação com os âmbitos da vida (GDSIs), ocorrência e intensidade do total de eventos estressores (IEE) com o teste t de Student, a fim de verificar diferenças nas médias com relação à variável contexto territorial. Por último, realizamos três regressões múltiplas com o indicador de bem-estar (SLSS) como variável dependente para amostra global, urbana e rural. Como variáveis independentes no modelo para amostra global introduzimos em um primeiro bloco as variáveis sociodemográficas (contexto territorial, gênero, ano escolar e idade), em um segundo, as variáveis: senso de comunidade e satisfação com os âmbitos da vida, e os eventos estressores cotidianos em um terceiro bloco. Nos modelos para amostras urbana e rural o procedimento se repetiu, com a única diferença que excluímos a variável contexto territorial do primeiro bloco em que foram introduzidas as variáveis sociodemográficas. Em cada bloco, o método de estimação utilizado foi o stepwise para selecionar a ordem das variáveis independentes. Os resultados foram analisados usando-se o software Statistical Package for the
Social Sciences (IBM SPSS - IBM Corp., Armonk, Estados Unidos), versão 19. O nível de confiança adotado nas análises foi de $95 \%$, o que equivale a um nível de significância $\mathrm{p}<0.0529$.

\section{Resultados}

Na análise dos estressores cotidianos para a amostra global observamos uma frequência média de ocorrência de 15,01 ( $\mathrm{DP}=6,68)$ eventos. A média da soma de escores de impacto para todos os eventos vivenciados foi de 48,01 ( $\mathrm{DP}=28,15$ ), com um intervalo entre 0 e 132 pontos, enquanto a média de impacto para cada evento foi de 1,59 (DP = 0,93), com um intervalo entre 0 e 4,40.

$\mathrm{Na}$ Tabela 1, apresentamos as maiores frequências de ocorrência e as maiores médias de intensidade atribuída, entre os trinta eventos estressores cotidianos analisados, considerando tanto a amostra global como as segmentações da amostra por contexto territorial, urbano e rural.

Na Tabela 2, observamos os oito eventos estressores cotidianos, dentre os trinta analisados, que apresentam diferenças significativas por meio de um teste de qui-quadrado nas porcentagens de ocorrência relacionadas ao contexto territorial. De maneira geral, vemos que no contexto urbano o porcentual de ocorrência dos eventos é maior para todos os estressores listados. Verificamos também que o evento ser assaltado na rua é o que maior diferença apresenta quando comparamos os contextos urbano $(41,8 \%)$ e rural (29,8\%), e que sofrer castigos e punições é o que menor diferença apresenta entre os dois contextos, urbano $(46,6 \%)$ e rural $(38,9 \%)$.

Ainda na Tabela 2, apresentamos uma análise comparativa das médias de intensidade atribuída ao evento estressor por contexto territorial utilizando o teste t de Student. Listamos os seis eventos estressores cotidianos, dentre os trinta analisados, que apresentam diferenças significativas entre os participantes das zonas urbanas e rurais. Novamente vemos que para os seis eventos, as crianças e adolescentes da zona urbana atribuem uma maior intensidade ao estressor vivenciado quando comparados aos da zona rural. O evento não poder utilizar computador, internet, videogame ou celular é o que maior diferença apresenta na intensidade atribuída quando comparado por contexto territorial, urbano $(\mathrm{m}=$ $2,59)$ e rural $(m=2,05)$. O evento que menor diferença de intensidade apresenta entre urbano $(\mathrm{m}=1,52)$ e rural $(\mathrm{m}=1,14)$ é novamente sofrer castigos e punições.

Na Tabela 3, encontramos as diferenças por contexto territorial com base no teste $\mathrm{t}$ de Student entre as médias: SLSS, SCI, GDSI(s) e total 
Tabela 1

Eventos estressores cotidianos com maior ocorrência e intensidade para amostra: global, urbana, rural.

\begin{tabular}{|c|c|c|c|c|}
\hline \multirow{2}{*}{$\begin{array}{l}\text { Frequências de ocorrência } \\
\text { Amostra global [ } n=757]\end{array}$} & \multirow[b]{2}{*}{$\%$} & \multicolumn{3}{|c|}{ Médias de intensidade atribuída } \\
\hline & & Amostra global $[n=757]$ & Média & DP \\
\hline Estar doente & 74,6 & Não poder utilizar internet, computador, celular & 2,40 & 2,14 \\
\hline Ter provas na escola & 69,4 & Estar doente & 2,18 & 1,80 \\
\hline Não poder utilizar internet, computador, celular & 64,5 & Terminar uma relação de namoro ou amizade & 2,11 & 2,01 \\
\hline Brigar com os irmãos & 64,4 & Ter provas na escola & 2,03 & 1,75 \\
\hline Terminar uma relação de namoro ou amizade & 63,4 & Ter pais que estão separados & 1,98 & 2,22 \\
\hline Ter alguém na família doente & 61,7 & Brigar com os irmãos & 1,97 & 1,86 \\
\hline Brigar com colegas na escola & 59,5 & Brigar com colegas na escola & 1,93 & 1,95 \\
\hline Ter de obedecer às ordens dos pais & 58,3 & Ter de obedecer às ordens dos pais & 1,91 & 2,00 \\
\hline Não ter dinheiro para comprar o que quer & 58,1 & Não ter dinheiro para comprar o que quer & 1,82 & 1,92 \\
\hline Não ter tempo para fazer o que gosta & 57,7 & Não receber cuidado nem atenção dos pais & 1,76 & 2,02 \\
\hline Amostra urbana [ $n=495]$ & $\%$ & Amostra urbana $[n=495$ ) & Média & DP \\
\hline Estar doente & 74,3 & Não poder utilizar internet, computador, celular & 2,59 & 2,14 \\
\hline Ter provas na escola & 72,4 & Ter provas na escola & 2,19 & 1,78 \\
\hline Não poder utilizar internet, computador, celular & 68,3 & Terminar uma relação de namoro ou amizade & 2,14 & 2,02 \\
\hline Brigar com os irmãos & 65,0 & Estar doente & 2,13 & 1,79 \\
\hline Terminar uma relação de namoro ou amizade & 64,5 & Brigar com os irmãos & 2,05 & 1,93 \\
\hline Amostra rural $[n=262]$ & $\%$ & Amostra rural $[n=262]$ & Média & DP \\
\hline Estar doente & 75,1 & Estar doente & 2,26 & 1,79 \\
\hline Ter provas na escola & 63,7 & Terminar uma relação de namoro ou amizade & 2,05 & 1,99 \\
\hline Brigar com os irmãos & 63,4 & Não poder utilizar internet, computador, celular & 2,05 & 2,10 \\
\hline Terminar uma relação de namoro ou amizade & 61,3 & Brigar com colegas na escola & 1,88 & 1,91 \\
\hline Não ter tempo para fazer o que gosta & 60,2 & Ter pais que estão separados & 1,87 & 2,16 \\
\hline
\end{tabular}

DP: desvio padrão.

de eventos estressores vivenciados, tanto para ocorrência como para intensidade. Vemos que as médias da escala de bem-estar e dos índices de satisfação com a vida: pessoal, escola, uso do tempo e saúde não apresentam diferenças significativas entre os participantes das zonas urbanas e rurais. Contudo, quando observamos as pontuações de satisfação com os âmbitos familiar, material, relacional e comunitário, constatamos que as crianças e adolescentes das zonas urbanas pontuam mais baixo na satisfação com quatro âmbitos citados. De todos os âmbitos investigados, a satisfação com o bairro ou zona onde vive é o que as crianças e os adolescentes encontram-se mais insatisfeitos. Verificamos ainda uma diferença significativa entre entorno urbano e rural no índice global de satisfação com os âmbitos da vida, em que novamente os participantes da zona urbana pontuam médias mais baixas. Vemos também que as maiores médias na escala senso de comunidade estão entre os alunos das zonas rurais e que os alunos das zonas urbanas pontuam níveis mais altos no total de eventos estressores cotidianos, tanto relacionado à ocorrência como a intensidade atribuída ao estressor.

Na Tabela 4, apresentamos três regressões lineares múltiplas tendo a escala de bem-estar SLSS como variável dependente para amostra global, urbana e rural. Observamos que nos três modelos, o senso de comunidade tem o maior coeficiente de regressão padronizado $(\beta)$, sendo uma importante variável associada ao bem-estar subjetivo da população estudada, sobretudo para crianças e adolescentes das zonas rurais. No que diz respeito aos âmbitos de satisfação com a vida, constatamos que nos três modelos os âmbitos pessoal e material são os que estão mais associados ao bem-estar da população pesquisada. Nos modelos para a amostra global e amostra urbana, a satisfação pessoal tem maior peso que a material. No caso do modelo para amostra rural a satisfação com bens materiais apresenta um $\beta$ 
Tabela 2

Diferenças por contexto territorial: eventos estressores cotidianos (frequências e médias).

\begin{tabular}{|c|c|c|c|c|c|c|c|c|c|}
\hline Ocorrência dos eventos estressores & \multicolumn{2}{|c|}{ Urbano (\%) [n = 495] } & \multicolumn{2}{|c|}{ Rural (\%) [n = 262] } & \multicolumn{2}{|c|}{ Total (\%) [n = 757] } & $\chi^{2}$ & Valor de $p$ & $\mathbf{v}$ \\
\hline Ter provas na escola & \multicolumn{2}{|c|}{72,4} & \multicolumn{2}{|c|}{63,7} & \multicolumn{2}{|c|}{69,4} & 5,974 & 0,015 & 0,089 \\
\hline Não poder utilizar Internet, computador, celular & \multicolumn{2}{|c|}{68,3} & \multicolumn{2}{|c|}{57,3} & \multicolumn{2}{|c|}{64,5} & 9,100 & 0,003 & 0,110 \\
\hline Ter de obedecer às ordens dos pais & \multicolumn{2}{|c|}{61,3} & \multicolumn{2}{|c|}{52,7} & \multicolumn{2}{|c|}{58,3} & 5,288 & 0,021 & 0,084 \\
\hline Sofrer castigos e punições & \multicolumn{2}{|c|}{46,6} & \multicolumn{2}{|c|}{38,9} & \multicolumn{2}{|c|}{43,9} & 4,044 & 0,044 & 0,073 \\
\hline Um dos pais ter de ir morar longe & \multicolumn{2}{|c|}{44,5} & \multicolumn{2}{|c|}{35,5} & \multicolumn{2}{|c|}{41,4} & 5,765 & 0,016 & 0,87 \\
\hline Mudar de casa, de bairro ou de cidade & \multicolumn{2}{|c|}{44,0} & \multicolumn{2}{|c|}{35,9} & \multicolumn{2}{|c|}{41,2} & 4,711 & 0,030 & 0,079 \\
\hline Ser assaltado na rua & \multicolumn{2}{|c|}{41,8} & \multicolumn{2}{|c|}{29,8} & \multicolumn{2}{|c|}{37,6} & 10,592 & 0,001 & 0,118 \\
\hline Ser suspenso na escola & \multicolumn{2}{|c|}{39,6} & \multicolumn{2}{|c|}{30,5} & \multicolumn{2}{|c|}{36,5} & 6,072 & 0,014 & 0,090 \\
\hline \multirow[t]{2}{*}{ Intensidade dos eventos estressores } & \multicolumn{2}{|c|}{ Urbano [n = 495] } & \multicolumn{2}{|c|}{ Rural [n = 262] } & \multicolumn{2}{|c|}{ Total [n = 757] } & \multirow[t]{2}{*}{$t$} & \multirow[t]{2}{*}{ Valor de $p$} & \multirow[t]{2}{*}{$d$} \\
\hline & Média & $\mathrm{DP}$ & Média & DP & Média & DP & & & \\
\hline Não poder utilizar Internet, computador, celular & 2,59 & 2,14 & 2,05 & 2,10 & 2,40 & 2,14 & 3,30 & 0,001 & 0,255 \\
\hline Ter provas na escola & 2,19 & 1,78 & 1,74 & 1,64 & 2,03 & 1,75 & 3,35 & 0,001 & 0,263 \\
\hline Um dos pais ter de ir morar longe & 1,65 & 2,10 & 1,25 & 1,91 & 1,51 & 2,04 & 2,59 & 0,010 & 0,199 \\
\hline Ser assaltado na rua & 1,57 & 2,08 & 1,10 & 1,87 & 1,41 & 2,02 & 3,17 & 0,002 & 0,238 \\
\hline Sofrer castigos e punições & 1,52 & 1,94 & 1,14 & 1,65 & 1,39 & 1,86 & 2,83 & 0,005 & 0,211 \\
\hline Ser suspenso na escola & 1,36 & 1,95 & 0,96 & 1,68 & 1,22 & 1,87 & 2,91 & 0,004 & 0,220 \\
\hline
\end{tabular}

Nota: dos 30 estressores analisados, mostramos aqueles itens que apresentam diferenças estatísticas significativas $(p<0,05)$ entre o contexto territorial: urbano e rural.

DP: desvio padrão.

Tabela 3

Diferenças por contexto territorial: Escala De Bem-Estar, Índices de Satisfação por Âmbitos Da Vida, Índice de Senso de Comunidade e ocorrência/intensidade atribuída dos eventos estressores (médias).

\begin{tabular}{|c|c|c|c|c|c|c|c|}
\hline \multirow[t]{2}{*}{ Indicadores } & \multicolumn{2}{|c|}{ Urbano [n = 495] } & \multicolumn{2}{|c|}{ Rural [n = 262] } & \multirow[t]{2}{*}{$t$} & \multirow[t]{2}{*}{ Valor de $p$} & \multirow[t]{2}{*}{$d$} \\
\hline & Média & DP & Média & DP & & & \\
\hline Escala: bem-estar subjetivo (SLSS - 0-4) & 2,74 & 1,00 & 2,74 & 0,93 & $-0,01$ & ns. & 0,000 \\
\hline Escala: senso de comunidade (0-4) & 1,91 & 0,92 & 2,25 & 0,85 & $-4,96$ & $<0,001$ & 0,384 \\
\hline Satisfação global por âmbitos da vida & 8,51 & 1,08 & 8,75 & 1,02 & $-2,96$ & 0,003 & 0,228 \\
\hline Satisfação âmbito: família & 9,05 & 1,16 & 9,24 & 1,11 & $-2,25$ & 0,025 & 0,167 \\
\hline Satisfação âmbito: bens materiais & 9,10 & 1,66 & 9,45 & 1,33 & $-3,14$ & 0,002 & 0,233 \\
\hline Satisfação âmbito: relações interpessoais & 8,37 & 1,60 & 8,69 & 1,53 & $-2,64$ & 0,009 & 0,204 \\
\hline Satisfação âmbito: bairro/zona onde vive & 6,72 & 2,30 & 7,25 & 2,14 & $-3,06$ & 0,002 & 0,238 \\
\hline Satisfação âmbito: saúde & 8,73 & 1,64 & 8,93 & 1,56 & $-1,65$ & ns. & 0,125 \\
\hline Satisfação âmbito: tempo & 9,05 & 1,55 & 9,06 & 1,55 & $-0,02$ & ns. & 0,006 \\
\hline Satisfação âmbito: escola & 8,46 & 1,21 & 8,63 & 1,33 & $-1,72$ & ns. & 0,134 \\
\hline Satisfação âmbito: pessoal & 8,60 & 1,31 & 8,74 & 1,10 & $-1,48$ & ns. & 0,116 \\
\hline Ocorrência total de eventos estressores & 15,51 & 6,67 & 14,07 & 6,63 & 2,78 & 0,006 & 0,216 \\
\hline Intensidade total de eventos estressores & 50,16 & 29,19 & 43,88 & 25,61 & 2,89 & 0,004 & 0,229 \\
\hline
\end{tabular}

DP: desvio padrão; ns.: não significativo.

mais elevado que a satisfação pessoal. No que diz respeito às variáveis independentes relacionadas aos eventos estressores cotidianos, observamos que no modelo para amostra global a intensidade do evento ser suspenso na escola entra com peso positivo, seguido da ocorrência dos estressores ter de obedecer às ordens dos pais e ter de mudar de escola, que aparecem com pesos negativos. No 
Análise de regressão linear múltipla com Escala de Satisfação com a Vida para Estudantes (SLSS).

\begin{tabular}{|c|c|c|c|}
\hline Amostra global [n = 757] & $\beta$ & $t$ & Valor de $p$ \\
\hline Senso de comunidade & 0,350 & 11,427 & $<0,001$ \\
\hline GDSI: pessoal & 0,253 & 7,212 & $<0,001$ \\
\hline GDSI: material & 5,180 & 5,294 & $<0,001$ \\
\hline Ter de obedecer às ordens dos pais (ocorrência) & $-0,084$ & $-2,844$ & 0,005 \\
\hline Ser suspenso na escola (intensidade) & 0,105 & 3,455 & 0,001 \\
\hline Ter de mudar de escola (ocorrência) & $-0,081$ & $-2,683$ & 0,007 \\
\hline Variável dependente: SLSS & $F=76,83$ & $R^{2}=0,383$ & $F^{2}=0,621$ \\
\hline Amostra zona urbana [ $n=495]$ & $\beta$ & $t$ & Valor de $p$ \\
\hline GDSI: pessoal & 0,304 & 7,069 & $<0,001$ \\
\hline Senso de comunidade & 0,321 & 8,575 & $<0,001$ \\
\hline GDSI: material & 0,161 & 3,878 & $<0,001$ \\
\hline Brigar com colegas na escola (ocorrência) & $-0,118$ & $-3,227$ & 0,001 \\
\hline Ser suspenso na escola (intensidade) & 0,106 & 2,760 & 0,006 \\
\hline Não ter tempo livre pra fazer o que gosta (ocorrência) & $-0,090$ & $-2,449$ & 0,015 \\
\hline Variável dependente: SLSS & $F=46,82$ & $\mathrm{R}^{2}=0,399$ & $F^{2}=0,664$ \\
\hline Amostra zona rural $[n=262]$ & $\beta$ & $t$ & Valor de $p$ \\
\hline Senso de comunidade & 0,435 & 8,326 & $<0,001$ \\
\hline GDSI: material & 0,205 & 3,667 & $<0,001$ \\
\hline Ter de obedecer às ordens dos pais (ocorrência) & $-0,154$ & $-3,004$ & 0,003 \\
\hline Ser assaltado na rua (ocorrência) & 0,103 & 2,036 & 0,043 \\
\hline GDSI: pessoal & 0,115 & 1,997 & 0,047 \\
\hline Variável dependente: SLSS & $F=29,81$ & $R^{2}=0,361$ & $F^{2}=0,565$ \\
\hline
\end{tabular}

GDSI: Índice de Satisfação por Âmbitos da Vida (General Domain Satisfaction Index).

modelo para amostra urbana, a ocorrência dos estressores brigar com colegas na escola e não ter tempo livre para fazer o que gosta aparecem com pesos negativos, e a intensidade do evento ser suspenso na escola aparece novamente com peso positivo. Para a amostra rural entram no modelo os eventos ter de obedecer às ordens dos pais e ser assaltado na rua, sendo o primeiro com peso negativo e o segundo com peso positivo. Os porcentuais de variância explicada $\left(\mathrm{R}^{2}\right)$ nos três modelos são: amostra global (38,3\%), amostra urbana $(39,9 \%)$ e amostra rural $(36,1 \%)$.

\section{Discussão}

Os resultados deste trabalho mostram o contexto de adversidade vivenciado pelas crianças e adolescentes estudantes de escolas públicas no Estado do Ceará. De um total de 30 estressores cotidianos analisados, a média vivenciada para a amostra global é de 15,01 eventos, havendo
$45,6 \%$ da amostra que está exposta a mais de quinze estressores em seu cotidiano; e dentre eles, $22,3 \%$ que vivencia cotidianamente mais de vinte eventos, do total de trinta estressores estudados. Quanto à intensidade atribuída, encontramos uma média para a amostra global de 1,59 por evento (em uma escala de 0-5), o que é esperado, dado que a literatura afirma que estressores cotidianos têm maior incidência e menor impacto quando comparados a estressores vitais. Contudo, é preciso enfatizar que existem eventos que apesar de cotidianos apresentam uma média de intensidade atribuída relativamente alta, como é o caso dos estressores: não poder utilizar computador, internet, videogame ou celular; estar doente; terminar uma relação de namoro ou amizade e ter provas na escola, todos com médias superiores a dois pontos. Também é importante considerar que os nove eventos estressores com maiores frequências de ocorrência são igualmente os que apresentam maior intensidade, mudando apenas a ordem atribuída. Assim, os 
eventos: estar doente; ter provas na escola; não poder utilizar computador, internet, videogame ou celular; terminar uma relação de namoro ou amizade; brigar com os irmãos, brigar com colegas na escola; ter de obedecer às ordens dos pais; não ter dinheiro para comprar o que quer; e não ter tempo para fazer o que gosta têm alta frequência e intensidade no cotidiano de crianças e adolescentes, tanto das zonas urbanas como das zonas rurais. Pesquisas anteriores sobre estressores cotidianos e vitais com crianças e adolescente vulneráveis de escolas públicas estaduais no Sul do Brasil também encontraram alta incidência de estressores cotidianos relacionados a: ter provas no colégio, ter de obedecer aos pais, brigar com irmãos e discutir com colegas 9,27.

Quando segmentamos a amostra e analisamos os cinco estressores que ocorrem com maior frequência entre os participantes de zonas urbanas e rurais separadamente, vemos que quatro deles se repetem mudando apenas a ordem de prevalência. Enfatizamos que na infância, eventos como: doenças, avaliações na escola, relações conflitivas com irmãos e rompimento de relações de namoro ou amizade estão presentes em ambos os contextos rurais e urbanos e são descritos pelas crianças e adolescentes como frequentes e estressantes, sendo necessário, portanto, suporte socioafetivo para superá-los. No que diz respeito a diferenças territoriais, constatamos que enquanto os alunos de escolas públicas da zona urbana citam o evento estressor não poder utilizar computador, internet, videogame ou celular como o terceiro mais vivenciado por eles, dentre os cinco de maior ocorrência, os alunos da zona rural descrevem não ter tempo para fazer o que gosta como o quinto evento estressor que mais vivenciam. Parece que a falta de acesso a recursos tecnológicos afeta mais as crianças e adolescentes que crescem nas cidades, sendo os que crescem em entornos rurais mais sensíveis à falta de tempo livre, por vezes preenchido pelo trabalho infantil e as responsabilidades precoces, como cuidar da casa ou dos irmãos menores. Estudos sobre o uso do tempo livre com alunos de escolas públicas em bairros de baixo nível socioeconômico no Sul do Brasil encontraram que as atividades domésticas ocupam 5\% do tempo diário destes adolescentes 30 .

No que diz respeito à intensidade atribuída, há dois eventos dentre os cinco de maior intensidade, que se diferenciam entre os participantes das zonas urbanas e rurais. Os alunos de zonas urbanas relatam que exigências escolares (ter provas na escola) e conflitos familiares (brigar com os irmãos) estão em terceiro e quinto lugares entre os estressores de maior intensidade; e os alunos das zonas rurais consideram que brigar com colegas na escola e ter pais que estão separados ocupam a quarta e quinta posições entre os estressores de maior intensidade, atribuindo importância às questões relacionadas à socialização. Ambos coincidem atribuindo aos estressores vividos nos âmbitos familiar e escolar maior intensidade.

Análises das diferenças significativas por contexto territorial demostram que as crianças e adolescentes da zona urbana estão mais expostos ao estresse cotidiano, quando comparados aos da zona rural; tanto no que diz respeito à ocorrência como para intensidade atribuída aos estressores. Há dois eventos em particular, a ocorrência de ser assaltado na rua e a intensidade de não poder utilizar computador, internet, videogame ou celular, que atingem significativamente mais aos alunos da zona urbana, contexto este marcado pela socialização por meio do uso da tecnologia e presença de violência nos espaços públicos.

A análise do bem-estar subjetivo quando avaliado usando-se uma escala livre de contexto com perguntas sobre satisfação com a vida em geral e considerando a variável contexto territorial, não apresenta diferenças significativas entre as médias, o que levaria a crer que o tipo de contexto de desenvolvimento ou a exposição a eventos estressores cotidianos não estaria associado ao bem-estar. Diferentemente de uma investigação portuguesa sobre assimetrias regionais no estilo de vida e satisfação de adolescentes, que encontrou níveis mais altos de bem-estar subjetivo naqueles que vivem em zonas urbanas 31 . Em nosso estudo, quando avaliamos a satisfação dos alunos das zonas urbanas e rurais por âmbitos específicos da vida, encontramos diferenças significativas em quatro dos oito âmbitos estudados. Os participantes pertencentes a entornos urbanos pontuam níveis mais baixos de satisfação com $a$ família, os bens material, as relações interpessoais e o bairro ou zona onde vivem. Ao mesmo tempo, pontuam valores mais altos na soma total dos trinta eventos estressores cotidianos, tanto para ocorrência como para intensidade atribuída. Recordando que os eventos analisados ocorrem nos âmbitos familiar, escolar, comunitário e pessoal. É necessário ainda enfatizar que de todos os âmbitos investigados, a satisfação com a zona onde vive é o que apresenta menor média (sobretudo para os da zona urbana), ressaltando o descontentamento das crianças e adolescentes estudados com aspectos relacionados a: espaços públicos ao ar livre, transporte público, polícia local e bairro/zona em geral. Na Espanha, crianças de 12 anos que vivem em contextos territoriais semiurbanos são as que apresentam maior satisfação por âmbitos da vida. Aquelas que vivem em 
entornos rurais pontuam mais alto na satisfação com os bens materiais, relacionamentos e uso do tempo, enquanto as que vivem em zonas urbanas estão mais satisfeitas com $a$ casa, $a$ saúde, $a$ escola e o bairro onde vivem, sendo este último o âmbito que menor satisfação apresenta, coincidindo com os resultados encontrados no presente estudo 15. A satisfação geral e por âmbitos da vida também guarda relação com o senso de comunidade, em que uma vez mais, as crianças e adolescentes das zonas urbanas são os que apresentam menores pontuações. Uma pesquisa na Itália encontrou valores mais elevados e maior associação entre satisfação com a vida e de senso de comunidade em adultos que vivem em pequenas comunidades, quando comparados ao que vivem em pequenas e grandes cidades 20 .

Os resultados das regressões múltiplas realizadas para amostra global, urbana e rural tendo a escala de bem-estar SLSS como variável dependente apontam que o senso de comunidade, seguido da satisfação com os âmbitos pessoal e material, são as variáveis que mais estão associadas ao bem-estar subjetivo (dentre as variáveis independentes analisadas) das crianças e adolescentes estudados, tanto na zona urbana como na rural. Em Portugal, uma pesquisa com crianças e adolescentes em dois bairros distintos de Lisboa, encontrou uma associação entre satisfação de vida e senso de comunidade com coeficiente de correlação de Pearson superior a 0,60 16. Em nosso estudo, o fato da satisfação com os bens materiais ter maior peso no modelo para amostra rural pode ser explicado pela situação de pobreza ser maior entre os alunos de escolas públicas e rurais. A satisfação pessoal ter peso maior para a amostra urbana pode ser compreendido pelo fato deste índice ser composto por itens referentes à liberdade, oportunidades, satisfação com corpo e aparência, segurança, autoconfiança, aspectos que podem ser mais valorizados pelos adolescentes que se desenvolvem em entornos urbanos. Destacamos ainda diferenças quanto aos eventos estressores cotidianos que entram nos modelos de avaliação do bem-estar subjetivo das populações urbana e rural pesquisadas. Na zona urbana, estão presentes estressores vivenciados no âmbito escolar como é o caso da violência entre pares (brigas com colegas) ou de questões disciplinares aplicadas pela escola (suspensões por "mau comportamento"), além da ausência de tempo livre para fazer o que gosta. No entorno rural, a ausência de estressores vividos nos âmbitos familiar (ter de obedecer às ordens dos pais) e comunitário (ser assaltado na rua) parece ter maior importância na avaliação do bem-estar subjetivo das crianças e adolescentes estudados.
A relevância do presente estudo está em possibilitar: (i) identificar os principais eventos estressores presentes no cotidiano dos alunos de escolas públicas no Nordeste do Brasil, participantes da pesquisa; (ii) enfatizar a situação de vulnerabilidade, em especial das crianças e adolescentes que vivem em entornos urbanos desfavorecidos; (iii) apontar os âmbitos da vida em que as crianças e adolescentes de entornos urbanos e rurais encontram-se mais insatisfeitos; e (iv) identificar o senso de comunidade como uma importante variável associada ao bem-estar subjetivo da população pesquisada.

A identificação de determinantes sociais de saúde presentes na vida das crianças e adolescentes estudados nos permite pensar em intervenções sistêmicas nos âmbitos familiar, escolar e comunitário necessárias na construção de ambientes sociais saudáveis, por meio da prevenção dos eventos potencialmente estressantes, do fortalecimento do sentimento de comunidade e da promoção do bem-estar subjetivo das crianças e adolescentes.

Uma das limitações deste trabalho diz respeito à impossibilidade de realizarmos generalizações, uma vez que a amostra, apesar de ampla, não é representativa. O desenho transversal do estudo também nos impede de estabelecer relações causais entre as variáveis analisadas, possibilitando apenas identificar associações entre elas.

Destaca-se ainda a necessidade de trabalhos futuros com amostras ampliadas e diversificadas, que investiguem sobre os fatores de risco e proteção à qualidade de vida na infância, baseando-se em estudos longitudinais e qualitativos que permitam conhecer a evolução da exposição aos estressores, do sentimento de comunidade e do nível de bem-estar subjetivo, analisando ainda diferenças de gênero, idade e titularidade da escola, não contempladas neste estudo. 


\section{Colaboradores}

D. P. Abreu contribuiu na concepção e projeto, análise e interpretação dos dados, redação do artigo, aprovação final da versão a ser publicada, responsável por todos os aspectos do trabalho na garantia da exatidão e integridade de qualquer parte da obra. F. Viñas colaborou na análise e interpretação dos dados e aprovação final da versão a ser publicada. F. Casas e C. Montserrat contribuíram na revisão crítica relevante do conteúdo intelectual e aprovação final da versão a ser publicada. M. González-Carrasco participou na revisão e aprovação final da versão a ser publicada. S. C. Alcantara participou da redação do artigo e revisão crítica relevante do conteúdo intelectual.

\section{Referências}

1. Fondo de las Naciones Unidas para la Infancia. Niños y niñas en un mundo urbano. Estado mundial de la infancia 2012. http://www.unicef.org/spa nish/sowc2012/pdfs/SOWC-2012-Main-ReportLoRes-PDF_SP_01052012.pdf (acessado em 26/ Jun/2015)

2. Assis SG, Avanci J, Oliveira RVC. Desigualdades socioeconômicas e saúde mental infantil. Rev Saúde Pública 2009; 43 Suppl 1:92-100.

3. Sameroff AJ. Environmental risk factors in infancy. Pediatrics 1998; 102(5 Suppl E):1287-92.

4. Corraliza JA. La ciudad, escenario vulnerable. In Fernadez B, Vidal T, editores. Psicología de la ciudad. Debates sobre el espacio urbano. Barcelona: Editorial UOC; 2011. p. 173-8.

5. Compas BE. Stress and life events during childhood and adolescence. Clin Psychol Rev 1987; 7: 275-302.

6. Kanner AD, Coyne JC, Schaefer C, Lazarus RS Comparison or two modes of stress measurement: daily hassles and uplifts versus major life events. J Behav Med 1981; 4:1-39.

7. Sandín B. El estrés: un análisis basado en el papel de los factores sociales. Int J Clin Health Psychol 2003; 3:141-57.

8. Carvalho PD, Barros MVG, Lima RA, Santos CM, Mélo EM. Condutas de risco à saúde e indicadores de estresse psicossocial em adolescentes estudantes do Ensino Médio. Cad Saúde Pública 2011; 27:2095-105.

9. Kristensen CH, Dell'Aglio DD, Leon JS, D'Incao DB. Análise da frequência e do impacto de eventos estressores em uma amostra de adolescentes. Interação 2004; 8:45-55.

10. Comissão para os Determinantes Sociais da Saúde. Redução das desigualdades no período de uma geração: igualdade na saúde através de ação sobre os seus determinantes sociais: relatório final. http://whqlibdoc.who.int/publications / 2010/9789248563706_por.pdf (acessado em 26/ Jun/2015).

\section{Agradecimentos}

O presente trabalho foi realizado com apoio da Capes, [Bolsa de Doutorado Pleno no Exterior (BEX 0715.13.1)].

11. Whitehead M. The concepts and principles of equity and health. Copenhagen: World Health Organization; 2000.

12. Buss PM, Pellegrini Filho A. A saúde e seus determinantes sociais. Physis (Rio J.) 2007; 17:77-93.

13. Rodríguez-Marín J. Psicología social de la salud. Madrid: Síntesis Psicología; 2001.

14. The WHOQOL Group. The development of the World Health Organization quality of life assessment instrument (The WHOQOL). In: Orley J, Kuyken W, editors. Quality of life assessment: international perspectives. Heigelberg: Springer Verlag; 1994. p. 41-60.

15. Casas F, Bello A. Calidad de vida y bienestar infantil subjetivo en España ¿Qué afecta al bienestar de niños y niñas españoles de 1o de ESO? Madrid: Fondo de las Naciones Unidas para la Infancia; 2012.

16. Elvas S, Moniz MJV. Sentimento de comunidade, qualidade e satisfação de vida. Anál Psicol 2010; 28:451-64.

17. Alcantara SC, Abreu DP, Farias AA. Pessoas em situação de rua: das trajetórias de exclusão social aos processos emancipatórios de formação de consciência, identidade e sentimento de pertença. Rev Colomb Psicol 2015; 24:129-43.

18. McMillan D, Chavis D. Sense of community: a definition and theory. Am J Community Psychol 1986; 14:6-23.

19. Sarason S. The perception and conception of a community. In: Sarason S, editor. The psychological sense of community: prospects for a community psychology. San Francisco: Jossey-Bass; 1974. p. 130-60.

20. Prezza M, Constantini S. Sense of community and live satisfaction: investigation in three diferente territorial contexts. Journal of Community \& Applied Social Psychology 1998; 8:181-94.

21. Pretty G, Andrews L, Collet C. Exploring adolescents' sense of community and its relationship to loneliness. J Community Psychol 1994; 22:346-58. 
22. Instituto Brasileiro de Geografia e Estatística. Síntese de indicadores sociais: uma análise das condições de vida da população brasileira. ftp://ftp. ibge.gov.br/Indicadores_Sociais/Sintese_de_Indi cadores_Sociais_2012/SIS_2012.pdf (acessado em 05/Out/2015).

23. Huebner ES. Correlates of life satisfaction in children. School Psychology Quarterly 1991; 6:103-11.

24. Casas F, Alfaro J, Sarriera JC, Bedin L, Grigoras B, Baltatescu S, et al. El bienestar subjetivo en la infancia: estudio de la comparabilidad de 3 escalas psicométricas en 4 países de habla latina. Psicoperspectivas 2015; 14:6-18.

25. Chavis DM, Hogge JH, McMillan DW, Wandersman A. Sense of community through Brunswik`s lens: a fisrt look. J Community Psychol 1986; 14:24-40.

26. Sarriera JC, Strelhow MRW, Bedin LM, Moura Jr. JF, Rodrigues AL, Calza TZ. Adaptation of the Sense of Community Index for Brazilian children. Paidéia (Ribeirão Preto) 2015; 25:39-47.

27. Poletto M, Koller SH, Dell'aglio DD. Eventos estressores em crianças e adolescentes em situação de vulnerabilidade social de Porto Alegre. Ciênc Saúde Coletiva 2009; 14:455-66.

\begin{abstract}
The study's overall objective was to investigate the relationship between psychosocial stressors, sense of community, and subjective wellbeing in urban and rural schoolchildren in Northeast Brazil, focusing on differences according to territorial context. The sample consisted of 757 participants, 495 from urban schools and 262 from rural schools, enrolled in the 6th and 7 th grades (9 to 18 years of age) in 21 municipal and state public schools, of which 13 urban and 8 rural, in 7 municipalities (counties) in Ceará State, Brazil. The study instruments were inventory of stressful events, scale of life satisfaction for students, index of sense of community, and satisfaction indices by life domains (family, material goods, relations, neighborhood/zone, health, time, school, and personal). The results indicate that socioeconomically underprivileged public schoolchildren from urban areas are more exposed to daily stress and score lower on satisfaction in specific domains of life and on sense of community. This latter is an important indicator for evaluating wellbeing in this young population.
\end{abstract}

Life Change Events; Life Quality; Child; Adolescent
28. Schütz F, Sarriera J, Bedin L, Montserrat C. Subjective well-being of children in residential care: comparison between children in institutional care and children living with their families. Psicoperspectivas 2015; 14:19-30.

29. Fisher RA. The arrangement of field experiments. Journal of the Ministry of Agriculture of Great Britain 1926; (33):503-13.

30. Sarriera JC, Tatim DC, Coelho RPS, Büsker J. O uso do tempo livre por adolescentes de classe popular. Psicol Reflex Crit 2007; 20:361-7.

31. Paupério T, Corte-Real NJ, Dias CS, Fonseca AM. Assimetrias regionais. Que diferenças nos estilos de vida e na satisfação com a vida dos adolescentes? Um estudo realizado em alunos do 3 o ciclo do Ensino Básico em Portugal. Ciênc Saúde Coletiva 2015; 20:17-28.

\section{Resumen}

El objetivo general del estudio es investigar la relación existente entre eventos estresores cotidianos, sentido de comunidad y bienestar subjetivo, en alumnos de escuelas urbanas y rurales del nordeste de Brasil, centrándose en las diferencias por contexto territorial. La muestra está formada por 757 participantes, 495 alumnos de escuelas urbanas y 262 de escuelas rurales, del 6o y 70 año (9 a 18 años) de 21 escuelas públicas municipales y estatales, 13 urbanas y 8 rurales, de 7 municipios de Ceará, Brasil. Los instrumentos utilizados fueron: inventario de eventos estresores, escala de satisfacción con la vida para estudiantes, índice de sentido de comunidad y ocho índices de satisfacción por ámbitos de la vida (familia, bienes materiales, relaciones, barrio/zona, salud, tiempo, escuela, personal). Los resultados indican que los alumnos de las escuelas públicas (desfavorecidos socioeconómicamente) de contextos urbanos están más expuestos al estrés cotidiano, puntúan más bajo en satisfacción con ámbitos específicos de la vida y sentido de comunidad, siendo este último un indicador importante en la evaluación del bienestar de la población estudiada.

Acontecimientos que Cambian la Vida; Calidad de Vida; Niño; Adolescente
Recebido em 04/Ago/2015

Versão final reapresentada em 15/Out/2015

Aprovado em 07/Dez/2015 